\title{
Strates
}

STRATES Matériaux pour la recherche en sciences sociales

13 | 2007

Paysage urbain: genèse, représentations, enjeux contemporains

\section{Le paysage de la gentrification à Barcelone}

Hovig Ter Minassian

\section{OpenEdition}

Journals

Édition électronique

URL : http://journals.openedition.org/strates/6312

DOI : $10.4000 /$ strates.6312

ISSN : $1777-5442$

Éditeur

Laboratoire Ladyss

Édition imprimée

Date de publication : 31 décembre 2007

ISSN : 0768-8067

Référence électronique

Hovig Ter Minassian, "Le paysage de la gentrification à Barcelone », Strates [En ligne], 13 | 2007, mis en ligne le 09 novembre 2008, consulté le 08 septembre 2020. URL : http://journals.openedition.org/ strates/6312 ; DOI : https://doi.org/10.4000/strates.6312

Ce document a été généré automatiquement le 8 septembre 2020

Tous droits réservés 


\title{
Le paysage de la gentrification à Barcelone
}

\author{
Hovig Ter Minassian
}

1 En quoi la lecture du paysage urbain peut-elle nous aider à comprendre la ville ? Dans quelle mesure suppose-t-elle un outil d'analyse pertinent des relations entre la société et le milieu urbain? Pour tenter de répondre à ces interrogations, j'interrogerai le paysage urbain, pour ce qu'il permet d'appréhender les processus de gentrification. Cette réflexion s'appuiera principalement sur la mise au point d'une méthodologie d'analyse d'un corpus photographique, dans le cadre d'un travail de thèse mené depuis 2005 sur la gentrification dans le centre ancien de Barcelone ${ }^{1}$.

Dès les années 1960 l'étude de la gentrification a porté sur le cas de Londres et, à sa suite, sur celui des villes anglo-saxonnes, britanniques comme nord-américaines. La gentrification est définie comme un phénomène à la fois urbain et social marqué par la récupération et la réhabilitation par des classes moyennes d'un stock de logements dégradés, se substituant ainsi progressivement aux classes populaires qui y vivaient auparavant, ou bien provoquant le départ de ces dernières ${ }^{2}$. Devenu plus largement symptomatique de l'évolution des centres-villes, y compris européens, le phénomène présente cependant à Barcelone des aspects particuliers.

Il est d'abord relativement récent dans l'agglomération catalane. Selon certains auteurs espagnols, on ne peut guère parler de gentrification avant la décennie $1980^{3}$. Les études portant sur le cas de Barcelone sont encore relativement peu nombreuses, y compris dans la littérature catalane ou castillane ${ }^{4}$, et interrogent notamment la définition et le mode d'analyse de la gentrification. Enfin, le contexte urbain très particulier du centre de Barcelone, où se côtoient quartiers en pleine redynamisation et quartiers en dégradation, invite à s'interroger sur la coprésence de phénomènes contradictoires, dont le découpage statistique, notamment à l'échelle micro-urbaine, rend parfois imparfaitement compte, en l'absence d'études exhaustives ou de données suffisamment pertinentes. La lecture du paysage urbain semble alors pouvoir ouvrir de nouvelles pistes pour comprendre les mutations sociospatiales du centre de Barcelone. Elle peut 
mettre en évidence les disparités du quartier et ses spécificités à l'échelle de la ville. Il y aurait alors un sens à étudier de pair paysage urbain et gentrification.

Lecture paysagère de la gentrification : références théoriques

4 Depuis le début des années 1980, le centre ancien de Barcelone est l'objet d'un réinvestissement matériel et symbolique. D’un espace délaissé par les politiques municipales jusqu'à la fin des années 1970 à un espace réinvesti, la matérialité et l'image du centre sont en pleine transformation. Les enjeux de cette mutation peuvent être éclairés par l'approche du paysage proposée par Augustin Berque, autorisant à penser le paysage dans sa double dimension matérielle et symbolique, mais permettant aussi de comprendre l'importance de l'évolution du regard que porte la société urbaine sur le centre ancien de Barcelone ${ }^{5}$. A. Berque, la relation entre la sphère humaine et la sphère naturelle n'est pas fondée sur la dissociation, mais au contraire l'interpénétration, le tissage complexe d'interrelations à la fois matérielles et abstraites, objectives et subjectives, réelles et symboliques, se nourrissant l'une l'autre. Le milieu, dont le paysage constitue la dimension sensible et symbolique, devient alors l'expression de la relation entre l'environnement et la société. Cette relation n'existe donc que pour un lieu donné à une époque donnée. Elle peut en outre évoluer, comme à Barcelone où la politique urbaine évolue, mais aussi le regard que les populations ( $\mathrm{y}$ résidant ou non) portent sur le centre de Barcelone. Il devient alors possible d'explorer les processus de gentrification par l'analyse du paysage urbain, dans la mesure où le paysage urbain est à la fois matériel (un tissu spécifique, un type de bâti, une végétation méditerranéenne, qui contribuent à créer des ambiances ${ }^{6}$ particulières) et porteur de sens, de valeurs (l'héritage d'un quartier ouvrier, qui renvoie à un imaginaire collectif autant qu'à l'histoire de la ville, les nouvelles valeurs attribuées par une politique de rénovation forte). Mais comment lire le paysage urbain?

5 Au début des années 1970, Sylvie Rimbert fut la première à proposer une analyse sémiologique du "paysage urbain », en élaborant une série de critères pour sa lecture. D'après cet auteur, le paysage est un espace construit traduisant une certaine réalité 7 L'espace fait sens, un sens qui peut être décrypté à travers deux notions essentielles, celle de signe et celle d'échelle. S. Rimbert définit en effet le paysage urbain comme « un assemblage de formes dont chacune est porteuse de significations et ces dernières sont hiérarchisées. C'est ainsi que ces formes-signes émettent des signaux qui ne sont pas tous perçus au même niveau ${ }^{8}$ ». Cette approche tend à favoriser le regard du sujet plutôt que l'objet et propose une lecture de la ville par le paysage urbain, par la forme, rompant ainsi d'une certaine manière avec l'analyse géographique classique (morphologique et fonctionnelle) de la ville. D'après S. Rimbert, la forme dit la fonction (ancienne ou présente) mais aussi le sens, la signification, parce que le paysage est porteur de "signes culturels ou psychiques ${ }^{9}$ ", plus ou moins lisibles par les individus. Ce qui compte alors dans l'étude du paysage urbain n'est pas tant l'espace en lui-même, que la façon dont il est perçu, le regard que l'on porte sur lui :

Ce qui importe à l'homme de la rue n'est pas tant la réalité elle-même que l'attrait ou la répulsion qu'elle provoque, le souvenir qu'il en garde, l'attachement ou l'indifférence qu'elle lui cause. Seul l'environnement subjectif et affectif le frappe ${ }^{10}$. [Enfin, ce paysage urbain se lit à plusieurs échelles, avec, par exemple] une vue de piéton, surtout sensible à la ville historique, et une vue d'automobiliste, surtout sensible au temps de parcours ${ }^{11}$.

Les notions de signe et d'échelle permettent également à S. Rimbert de faire jouer les correspondances entre un paysage extérieur, des images mentales qui s'y projettent, et 
l'inconscient de l'observateur ${ }^{12}$. Si le paysage de Barcelone se donne à lire, sa lecture ne relève pas seulement des signes que le paysage transmet, mais également de l'état d'esprit de l'observateur. Il parait ainsi essentiel de comprendre dans quelle mesure la perception des mutations du centre ancien de Barcelone n'est pas la même selon qu'elle est vécue par exemple de l'intérieur, par les résidents, ou de l'extérieur, par les touristes, les décideurs, ou même l'investigateur ${ }^{13}$. La rénovation d'un centre-ville est un acte politique fort, qui peut bouleverser le tissu social. S. Rimbert invite donc à « descendre dans la rue » et identifier les messages plus ou moins implicites du paysage urbain, démarche qui sera adoptée dans l'étude du centre de Barcelone. Ces messages peuvent être d'ordre documentaire, apportant une information (le nom d'une rue, la direction d'un musée...); d'ordre conceptuel, révélant un ordre ou une structure (indiquant par exemple que l'on se trouve dans un centre-ville historique...) ; ou bien d'ordre affectif, faisant surgir des émotions, comme le plaisir, la curiosité, ou réveillant des souvenirs.

7 Si ce type d'analyse tend à privilégier le regard dans la lecture du paysage urbain, on peut cependant s'interroger sur une approche plus globalisante, qui tenterait de restituer le paysage urbain dans toute sa dimension plurisensorielle: visuelle, mais aussi auditive, olfactive. Barcelone est une grande métropole méditerranéenne, foyer important de l'immigration nationale et internationale. La proximité de la mer, du port, la végétation, les activités du centre, le brassage ethnique et culturel, contribuent à forger une ambiance particulière dans le centre ancien. Mais comment transcrire dans un texte cette dimension plurisensorielle et les sensations qui en découlent ? La dimension visuelle sera, elle, restituée par la photographie. Le rapport entre la géographie et la photographie a déjà fait l'objet de nombreuses analyses. En effet, le géographe utilise ce mode d'illustration dès le xix siècle. Pour Didier Mendibil, «le rapport aux images manifeste l'existence de systèmes iconographiques à travers lesquels s'exprime et se structure la fonction sociale de la géographie ${ }^{14}$ ». Autrement dit, l'acte photographique renvoie à des normes, un rapport à l'image formalisé, voire codifié. Ce rapport a pu évoluer dans le texte, par la place de l'image dans le corpus géographique, son usage, sa technique. D. Mendibil souligne que l'acte photographique et son inscription dans un travail de type géographique supposent généralement trois modes d'intervention, chacun codifié d'une certaine manière. Le premier mode d'intervention est l'acte photographique lui-même, c'est-à-dire la fabrication d'image et l'élaboration de points de vue, notamment par le choix du cadrage. Le second est la sélection des images qui serviront à illustrer le travail, ce qui comprend l'ensemble des opérations de classement, de tri, de mise en ordre, mais aussi de contextualisation et de mise en interrelation des images. Le troisième est le choix d'un procédé d'exposition au sein de l'œuvre, avec ses dispositifs variables: un titre, une légende, toutes formes de paratexte.

8 Ces trois interventions successives guident notre propre parcours photographique sur le terrain d'étude. Dans un souci d'objectivité, l'opération la plus délicate est sans doute celle de la sélection des images qui serviront par la suite de matériau à l'analyse. Le parcours photographique cherche à mettre en évidence la multiplicité des situations, des contextes. Quels aspects du phénomène étudié la photographie est-elle en mesure de traduire? Cette question est abordée par Jean-Marie Schaeffer, dans L'image précaire ${ }^{15}$. D'après l'auteur, la photographie est un "signe de réception ", c'est-à-dire qu'il ne propose pas de lecture conventionnelle, et se contente de délivrer une certaine 
information, à moins justement que la photographie ne tienne explicitement lieu de message. Il précise même qu'un "signe n'a d'existence que pour un interprétant au moins virtue ${ }^{16}$ ». Deux questions se posent alors. D'abord, qu'apporte l'analyse d'une photographie par rapport aux phénomènes étudiés; ensuite, sur quoi les choix photographiques doivent-ils s'appuyer: une connaissance préexistante du terrain d'étude qui fait préférer certains lieux plutôt que d'autres, la recherche de certaines composantes du paysage urbain, comme le type de commerces, l'usage de l'espace public... ? Cette connaissance préexistante, J.-M. Schaeffer l'appelle "savoir latéral», autrement dit ce que nous savons par ailleurs sur le sujet de la photographie. Cette information redondante (puisqu'elle est la fois présente dans notre mémoire et dans la photographie) est une clef de compréhension de certains types de photographie. En ce sens, dans le cadre de notre méthode, l'analyse du paysage urbain à travers un corpus photographique ne cherche pas à se substituer à l'analyse statistique, par exemple, mais à la prolonger.

La Rambla del Raval, une étude de la gentrification à l'échelle microlocale

9 L'étude suivante sur un secteur du centre ancien de Barcelone se veut une première mise à l'épreuve méthodologique de l'analyse des paysages urbains par la photographie.

10 La bibliographie sur la gentrification révèle que l'étude du phénomène a pu donner lieu à deux types d'approche. La première est fondée sur une analyse statistique et quantitative de la gentrification, en travaillant par exemple sur des données sociodémographiques, des données concernant la mobilité résidentielle ou encore sur les registres d'imposition. La seconde consiste en une approche plus qualitative, et s'appuie sur des entretiens, et plus rarement le parcours photographique. Aucune de ces deux approches ne semble épuiser complètement l'analyse de la gentrification à l'échelle micro-urbaine. L'intérêt est méthodologique et scientifique : les entretiens et les parcours photographiques rendent plus difficilement compte de l'ampleur numérique de la gentrification, de la hausse de la valeur foncière ou bien des changements socioéconomiques. Inversement, $d u$ fait des données statistiques disponibles, il est parfois malaisé de saisir les logiques socioculturelles qui sous-tendent la gentrification, cerner les parcours biographiques ou appréhender les effets de la gentrification sur le paysage urbain, notamment à l'échelle micro-urbaine. Comprendre la gentrification revient à lire un processus qui investit à la fois la composition sociodémographique et les représentations urbaines du centre de Barcelone, d'où peutêtre l'intérêt de mener de front approche statistique et lecture du paysage urbain. Le second enjeu de cette double approche relève du "savoir latéral », pour reprendre l'expression de J.-M. Schaeffer. Il s'agit, par l'approche statistique, de cibler les secteurs qui mériteraient de faire l'objet d'une analyse du paysage urbain, ou bien, inversement, de révéler par le parcours photographique des secteurs où les processus de gentrification ne sont pas encore mesurables statistiquement à l'échelle micro-urbaine.

11 Le «Raval » est l'un des quatre quartiers qui composent le district de "Ciutat Vella ${ }^{17}$ ». Il tient une place particulière dans la géographie de Barcelone, du fait de son histoire et de l'imaginaire urbain et social qu'il véhicule à l'échelle de la ville. Ancien faubourg de la Barcelone médiévale, il devient rapidement le réceptacle de l'immigration espagnole puis étrangère. Dès le XVIII ${ }^{e}$ siècle, le Raval accueille ateliers et fabriques textiles, ainsi que les activités portuaires. Cette époque correspond également à la première grande vague migratoire (essentiellement catalane) dans le centre ancien de Barcelone. 
L'industrialisation du XIX ${ }^{e}$ siècle marque profondément la physionomie du quartier, aussi bien dans sa dimension urbanistique que sociale. À partir du milieu du $\mathrm{xx}^{\mathrm{e}}$ siècle, le départ des industries vers la périphérie, la crise des activités traditionnelles, la lente dégradation du tissu urbain et des logements, mais aussi la déprise démographique ${ }^{18} \mathrm{du}$ quartier justifient dès la fin du franquisme une réflexion sur le devenir du Raval et la mise en place d'une politique de rénovation forte.

Les auteurs espagnols soulignent qu'il y a toujours eu urbanistiquement et sociologiquement deux Raval: le Raval nord, plus ouvrier et au tissu urbain plus aéré, et le Raval sud, davantage tourné vers le port et les activités de loisirs, mais aussi plus vulnérable à la criminalité ${ }^{19}$. C'est au sujet de cette partie du Raval qu'est né le mythe du Barrio Chino, dans le premier quart du $\mathrm{xx}^{\mathrm{e}}$ siècle, à la fois quartier des loisirs (avec ses cabarets, ses théâtres), des plaisirs (avec le développement de la prostitution), mais aussi des luttes sociales, et des criminels. Quartier populaire où les bourgeois de l'Eixample ${ }^{20}$ venaient s'encanailler, le Barrio Chino tient une place particulière dans l'imaginaire collectif de Barcelone, véhiculant les images d'un quartier dangereux, mais aussi vivant, populaire, animé. On comprend mieux, dès lors, les enjeux d'une politique de rénovation urbaine, entre amélioration des conditions d'habitabilité, volonté de réduire les espaces interlopes et de donner une image plus policée du centre, mais aussi perte du caractère populaire du centre ancien, de ses spécificités urbaines et sociales, ce qui finalement, pour beaucoup, semblaient faire le charme du Raval. Pourtant, comme le montre Sophie Savary dans sa thèse sur les paysages géolittéraires de Barcelone $^{21}$, le mythe du Barrio Chino perdure, au moins dans la littérature espagnole contemporaine et dans les guides touristiques. Les processus de gentrification et l'analyse du paysage urbain renvoient alors à la question de l'imaginaire collectif et de la transmission de la mémoire ${ }^{22}$. À travers la transformation du paysage urbain, quelles nouvelles images du Raval, de ses habitants et de ses activités, sont véhiculées par la politique de rénovation depuis les années 1980 ?

La « Rambla del Raval » se présente comme une grande voie occupée par un terre-plein central. Elle est un élément essentiel de la politique municipale de réhabilitation du centre, d'une part par son importance, la superficie concernée $(58 \mathrm{~m}$ de large pour $317 \mathrm{~m}$ de long) et le type de réaménagement proposé ; d'autre part par sa localisation au cœur du Raval. Ce qui frappe dans un premier temps, c'est le contraste des édifices, des habitants, des commerces, de l'ambiance. En ce sens, la Rambla del Raval peut apparaître comme un vecteur de la gentrification du centre ancien de Barcelone.

Le réaménagement de cette nouvelle Rambla est inaugurée en septembre 2000 et s'inscrit dans la politique plus large de rénovation du Raval, illustrée par de grandes opérations ${ }^{23}$, et par un important soutien financier à la réhabilitation privée. Modifier le tissu urbain du Raval implique une transformation des regards portés sur ce quartier. Avec les nouveaux équipements culturels et la redynamisation du marché immobilier, la municipalité cherche à donner une image transformée du Raval : celle d'un quartier foisonnant, dynamique, multiculturel et socialement mixte. C'est donc à la fois une nouvelle relation matérielle et un nouveau rapport subjectif qui s'instaure entre les Barcelonais (habitants du Raval ou non) et le cœur du Raval, bien qu'une partie du quartier reste fortement dégradée.

15 L'analyse statistique de ce secteur, à différentes échelles de découpage, renseigne sur son profil socioéconomique par rapport à celui du centre-ville et de Barcelone. Une première limite apparaît d'emblée dans l'étude des mutations urbaines à une échelle si 
fine : aucun découpage statistique ne concorde parfaitement avec l'espace étudié. Ainsi, à l'échelle des Zonas de Recerca Petita (ZRP) ${ }^{24}$ la Rambla del Raval se trouve à cheval sur 3 zones (nos 29, 30 et 32), et sur 6 secteurs à l'échelle des Seccíones Estadístiques (SE) ${ }^{25}$ $(105,106,107,108,116$ et 117), comme en témoigne la carte 1.

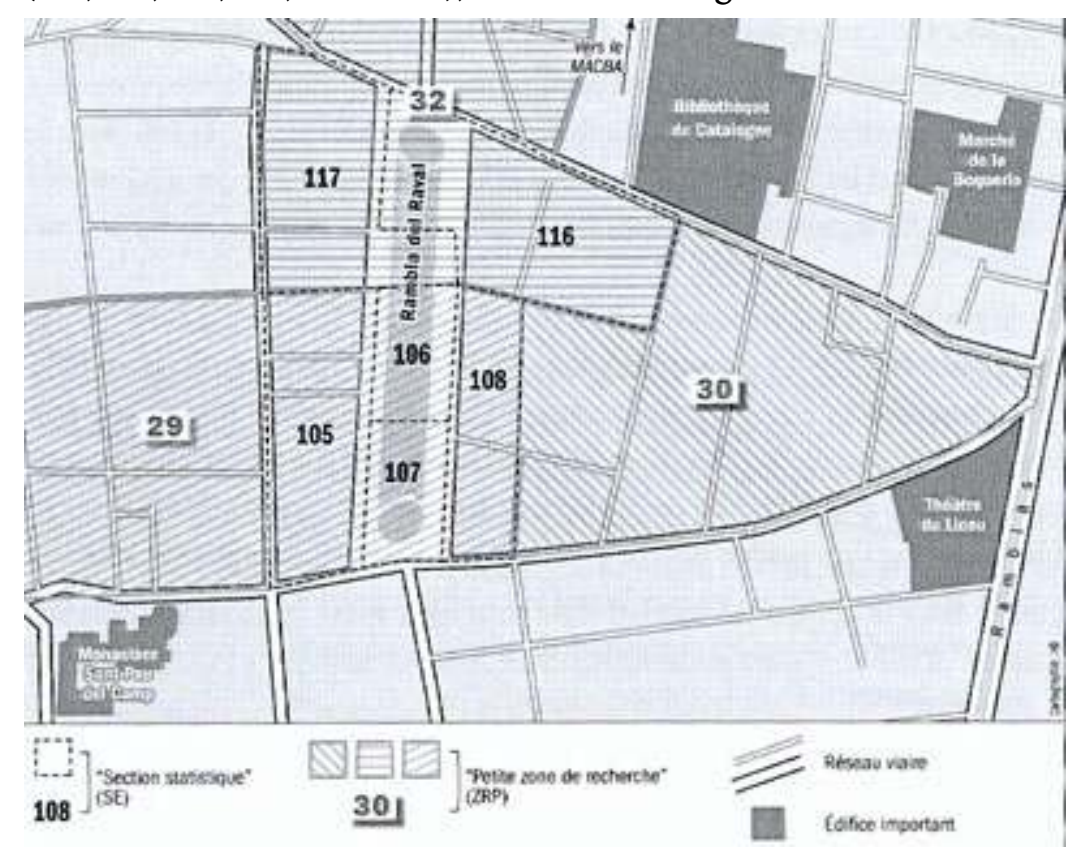

Carte 1. Fractionnement statistique de la Rambla del Raval.

On s'interrogera d'abord sur d'éventuelles correspondances entre les spécificités dégagées pour l'ensemble des zones statistiques concernées et celles du secteur étudié. Avant l'opération d'aménagement de la Rambla del Raval, 2 SE couvraient les îlots démolis par la suite. Après l'ouverture de la Rambla, la carte des SE a donc été réajustée, ce qui rend l'approche chronologique plus délicate. Néanmoins, on peut d'ores et déjà émettre un certain nombre de constats sur l'évolution sociodémographique du secteur.

S'il est malaisé de connaître précisément l'importance de la population concernée par l'opération de réaménagement de la Rambla del Raval (y compris ceux qui vivaient dans les logements qui ont dû être démolis), les 3 ZRP comptent en 2005 plus de 12200 habitants, nombre en augmentation depuis 2001, témoignant d'une inversion de tendance. Mais on peut difficilement affirmer que la population a augmenté ou baissé autour de la Rambla del Raval, d'autant que cette augmentation peut être due soit à l'arrivée de nouvelles populations, soit au redécoupage des zones statistiques. Cependant, du fait de la démolition de certains édifices, l'opération elle-même a provoqué le départ d'un grand nombre de familles ${ }^{26}$. Sur l'ensemble des SE, en 2005, on compte plus de 4600 habitants. C'est une population globalement plus jeune que celle de l'ensemble du Raval, ou de Ciutat Vella, district lui-même caractérisé par un fort vieillissement de la population. Les secteurs 105 et 108 possèdent une proportion de plus de 65 ans particulièrement faible par rapport au district (respectivement 9,0 et $6,7 \%$ contre 18,6\%). Les secteurs 106 et 107 ont, à l'inverse, des taux supérieurs à la moyenne, mais leur population étant très faible (respectivement 4 et 13 habitants), il est difficile d'en tirer des conclusions. La proportion de moins de 35 ans en 2005 est également partout supérieure à la moyenne de Ciutat Vella, voire supérieure à la 
moyenne des ZRP. Ainsi, elle est de 41,1\% pour le ZRP 29 (qui comprend les SE 103 à 107) alors que le secteur 105 compte $49,7 \%$ de moins de 35 ans. La différence est tout aussi importante pour la part des 20-35 ans en 2005, avec:

\begin{tabular}{|c|l|l|}
\hline & 1991 & 2005 \\
\hline \hline Barcelone & $7,35 \%$ & $18,83 \%$ \\
\hline \hline Ciutat Vella & $3,39 \%$ & $12,30 \%$ \\
\hline \hline Raval & $2,55 \%$ & $10,27 \%$ \\
\hline \hline ZRP 29 & $0,76 \%$ & $8,93 \%$ \\
\hline
\end{tabular}




\begin{tabular}{|l|l|l|}
\hline ZRP 30 & $1,80 \%$ & $11,20 \%$ \\
\hline \hline ZRP 32 & $1,50 \%$ & $10,04 \%$ \\
\hline
\end{tabular}

Source : Municipalité de Barcelone

suffisamment fine (comme la part de la population ayant un diplôme supérieur ou bien la catégorie socio-professionnelle à l'échelle des $\mathrm{SE}$ ) traduisent imparfaitement la réalité des processus de gentrification (élévation généralisée des conditions de vie ou bien substitution des catégories populaires par des populations plus aisées?) mais soulignent l'intérêt de ce secteur particulier comme terrain d'étude de l'analyse paysagère. Si les données statistiques pour le centre de Barcelone permettent d'appréhender un certain nombre de phénomènes, comme l'évolution générale des conditions de vie, les recompositions démographiques à une échelle plus ou moins grande, ou bien l'évolution du marché immobilier, leurs limites apparaissent en revanche assez vite pour étudier un secteur à une échelle d'analyse si fine, interroger les mutations du paysage urbain ou bien encore analyser les nouvelles pratiques spatiales de la Rambla del Raval. L'appréhension en termes de paysage pourrait ainsi affiner la réflexion et permettre de saisir la ville dans sa continuité à la fois spatiale (le tissu urbain) et temporelle (l'évolution d'un quartier porté par une politique très forte de réhabilitation). L'intérêt de la photographie peut apparaître alors à plusieurs niveaux. Elle met à distance et autorise des lectures ou relectures multiples, y compris loin du terrain d'étude. Elle permet la comparaison immédiate (entre un avant et un après ou bien deux espaces distants). Enfin, elle tend à privilégier le détail.

2 met en évidence le contraste entre litinéraire photographique tel qu'il a été effectué lors du travail de terrain, et l'ordre dans lequel les photographies sélectionnées ont été restituées dans le texte. 


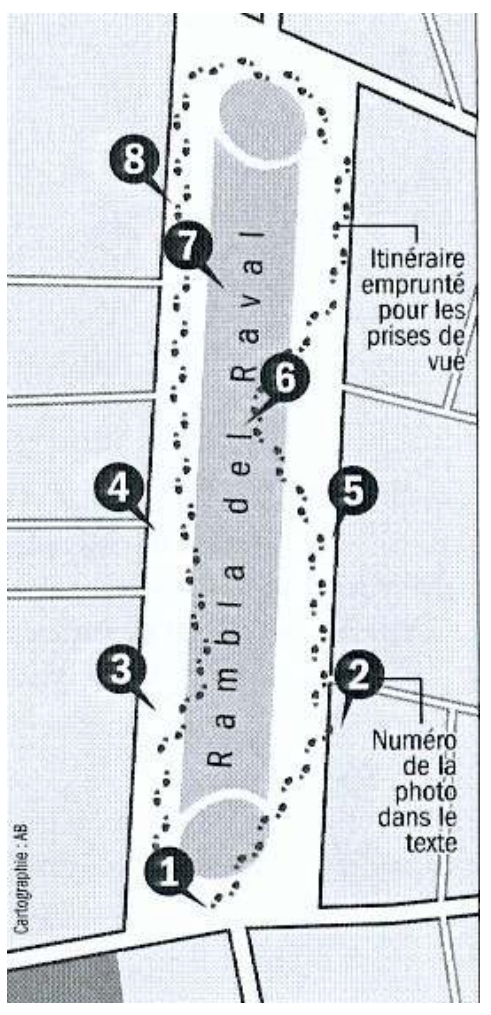

Carte 2. Itinéraire photographique.

Sur le terrain, le cheminement a été circulaire, en partant du sud vers le nord, par le côté est, puis en redescendant du nord vers le sud, par le côté ouest, afin de travailler de manière systématique sur les abords de la Rambla ${ }^{27}$. Dans le texte, les photographies privilégient au contraire le cheminement transversal, en alternant côté est et côté ouest, tout en progressant vers le nord. Cette différence explicite la constitution et la restitution du corpus photographique. Dans un premier temps, c'est la collecte d'information qui a été privilégiée. Elle correspond au premier mode d'intervention explicité par D. Mendibil : les différents choix de cadrage et l'élaboration des points de vue. Dans un second temps, l'opération de tri vise à démontrer une hypothèse de travail, celle d'une gentrification progressive des abords de la Rambla, par le nord et par l'est, c'est-à-dire les espaces les plus proches des zones touristiques et culturelles principales $\mathrm{du}$ centre ancien de Barcelone. Elle correspond au second mode d'intervention explicitée par D. Mendibil, c'est-à-dire l'ensemble des opérations de sélection et de classement.

Les abords de la Rambla del Raval ont fait l'objet d'importants travaux de réaménagement, qui ont donné la priorité au logement. La partie est de la Rambla est bordée de nouvelles constructions de logements, comme celui de la figure 1. C'est un immeuble relativement peu élevé $(R+5)$ : la préoccupation première a sans doute été d'intégrer au mieux ces nouveaux édifices au tissu urbain ancien. La présence de certains éléments (le parking à vélo, les doubles vitrages) témoigne sans doute d'un effort apporté au confort général des logements, mais aussi l'adaptation à un certain mode de vie. Circuler en voiture dans les ruelles étroites de Ciutat Vella n'est pas toujours évident, pas plus que de préserver le calme du logement lorsque les fenêtres de l'appartement donnent sur un espace public qui peut être très fréquenté, donc parfois bruyant, et souvent sollicité lors des différentes manifestations (fêtes, 
concerts...) qui rythment l'année ${ }^{28}$. Au pied du bâtiment, un magasin de vêtements a installé sa vitrine. Enfin, au premier plan, les palmiers apportent un peu d'ombre à la Rambla. Quelle image du quartier ancien ce bâtiment nous renvoie-t-il ? Ne favorise-t-il pas l'émergence d'une nouvelle image du centre, celle d'un quartier rajeuni, moderne, voire "normé29 ", qui joue parfois sur des stéréotypes, avec des palmiers censés rappeler la «méditerranéité » du lieu comme pour mieux l'ancrer dans le local ${ }^{30}$ ? Les nouvelles populations ou les nouveaux usages ne sont pas seuls à favoriser un nouveau regard sur le centre; le type de bâti, bien qu'il contraste brutalement avec l'architecture traditionnelle du centre ancien de Barcelone, y participe également.

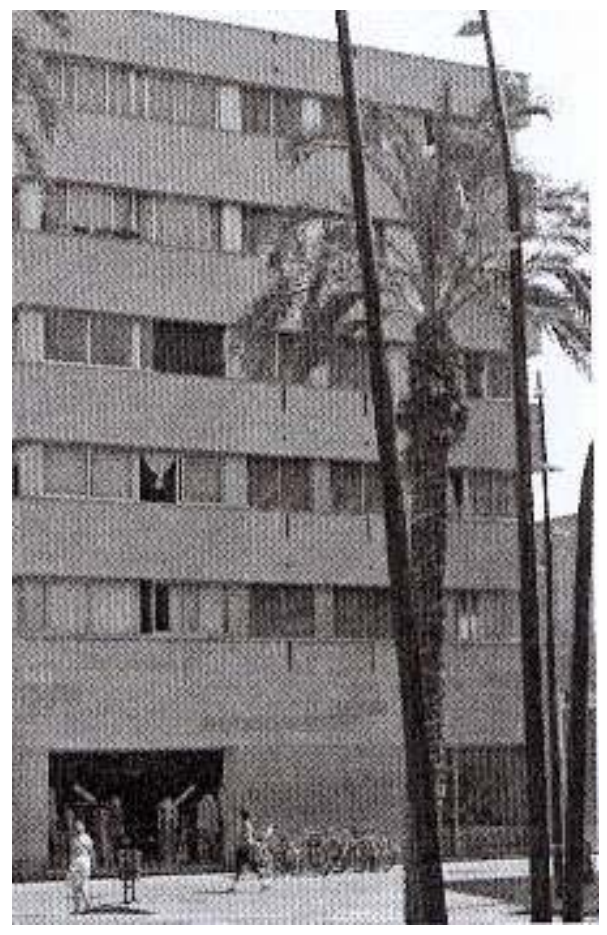

Figure 1 : Un immeuble récent sur la Rambla del Raval. Sept. 2006.

Le bâtiment de la figure 2 est situé un peu plus loin le long de la Rambla, côté est. Au premier plan, on distingue très bien les barrières d'un chantier de rénovation des réseaux souterrains. Sur la gauche, un vaste espace s'ouvre, en cours de terrassement, emplacement de futurs bâtiments de logements. Derrière le rideau d'arbres, un immeuble réhabilité et peu élevé $(R+4)$ occupe le second plan. Au rez-de-chaussée, une agence immobilière, spécialisée dans les transactions dans le centre ancien de Barcelone, a ouvert ses portes. La présence de cette dernière peut-elle alors traduire une réactivation du marché immobilier? A-t-elle un rôle potentiel de vecteur de la gentrification? Là encore, le paysage urbain se construit autour de valeurs liées à la rénovation, mais aussi à l'affirmation de l'individualité et de nouvelles pratiques de l'espace urbain qui passent par une certaine capacité économique. La politique de rénovation bouleverse la fonction mnémonique du paysage, notamment comme réceptacle de la mémoire collective. En effet, avant la restructuration du quartier, dans ce secteur se trouvait une fontaine publique. Comme l'a rappelé au cours d'un entretien la présidente d'une association de riverains ${ }^{31}$, cette fontaine fut un haut lieu de sociabilité, que les riverains ont tenté de sauver des pelleteuses mécaniques. La question se pose alors, de la mesure dans laquelle la transformation du paysage peut 
menacer la transmission de la mémoire collective et remettre en cause l'identification d'une population à un quartier; questionnement d'autant plus fondamental que l'on se trouve ici au cœur de l'ancien Barrio Chino chargé de mythes.

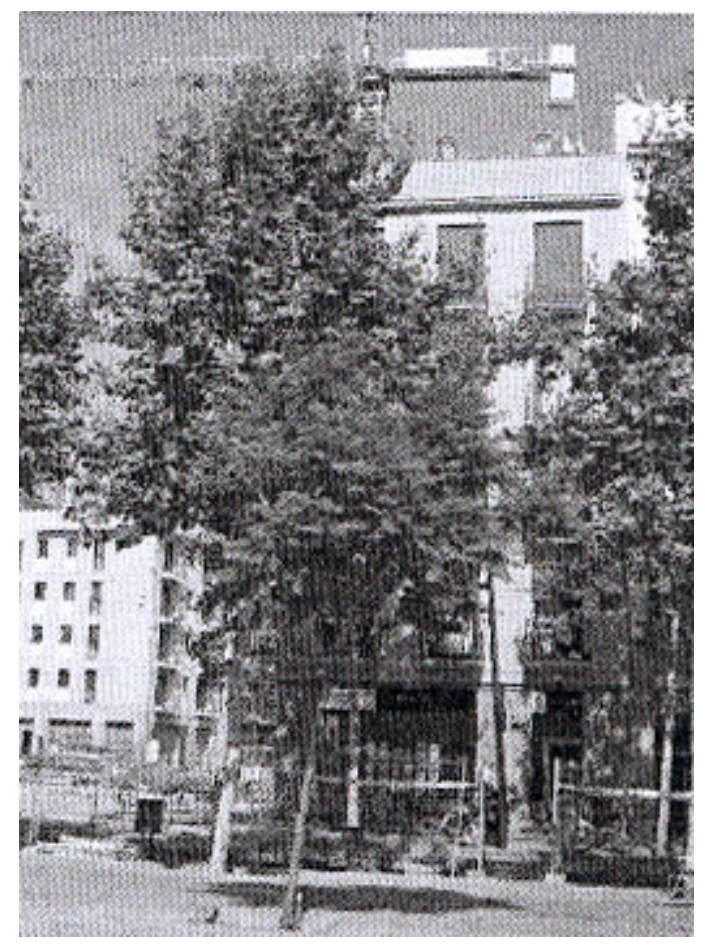

Figure 2. La dynamique immobilière de la Rambla del Raval. Sept. 2006.

À peu près en face du bâtiment précédent, de l'autre côté de la Rambla, le type de commerces présents diffère fortement. Le paysage sonore n'est pas non plus le même, puisqu'ici, c'est plutôt l'arabe, et non le castillan ou le catalan, que l'on entend. Le rezde-chaussée du bâtiment rénové est occupé à gauche par une boucherie halal, faisant également office de supérette. Sa devanture, écrite en castillan et en arabe, tient une double place dans la typologie des messages du paysage urbain dégagée par S. Rimbert. Message d'ordre documentaire et message d'ordre conceptuel, il témoigne de la forte composante migratoire mise en évidence par l'analyse statistique, confirmée ici par la présence de commerces de proximités ${ }^{32}$. Un certain type de commerces a tendance à se développer dans le Raval, à destination d'une population étrangère nombreuse (boutiques alimentaires spécifiques, services de télécommunication vers l'étranger, cybercafés). Participant du bouleversement du cadre de vie traditionnel des habitants, leur prolifération inquiète parfois les élus locaux ou les responsables d'associations de riverains. La figure 3 traduit donc la forte composante migratoire de la population du Raval et le maintien de commerces de proximité spécifiques qui lui sont destinés. Fautil y voir également une certaine résistance aux processus de gentrification? 


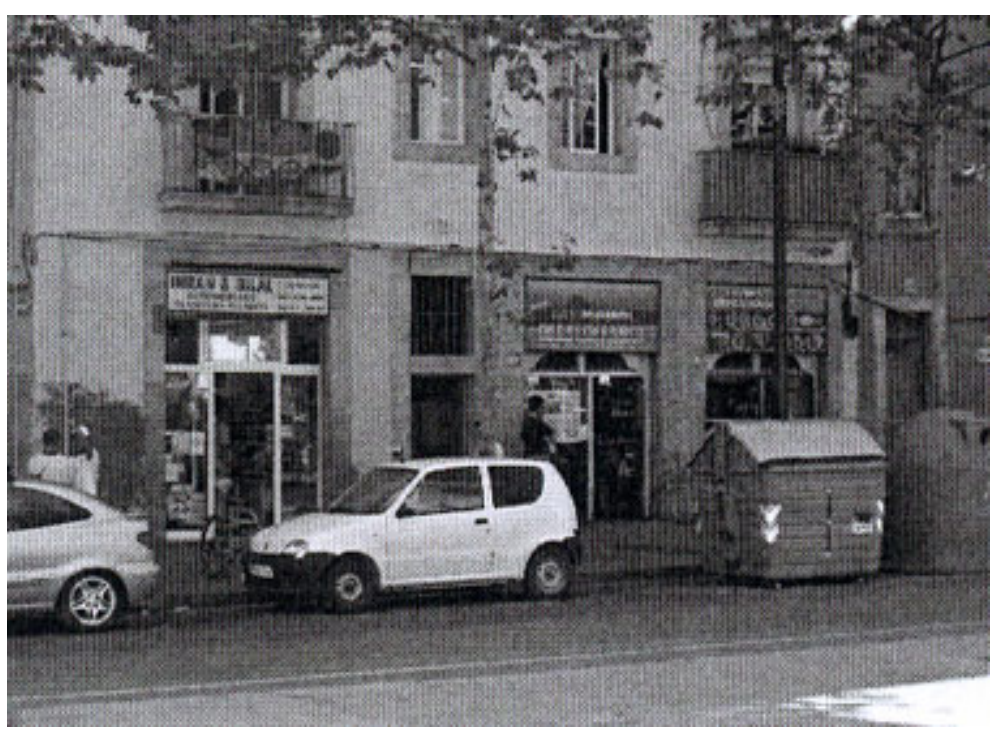

Figure 3. Un paysage commercial à forte composante migratoire. Sept. 2006.

31 On peut mettre en regard les figures 4 et 5 dans la mesure où elles participent de la diversité des formes d'intervention dans le centre en matière de logements. La figure 4 montre un immeuble qui n'a pas encore fait l'objet d'une récupération. La façade n'a pas été ravalée, les balcons n'ont pas été repeints, les stores sont assez sommaires. Au rez-de-chaussée la succession de rideaux de fer pourrait être l'indice de la disparition $\mathrm{du}$ petit commerce ${ }^{33}$. La figure 5 présente au premier plan une grande pancarte annonçant la construction future de nouveaux logements et au second plan un chantier. Il s'agit de la construction de 31 nouveaux logements de $\mathrm{HPO}^{34}$. L'installation d'une pancarte n'est pas anodine, mais bien symbolique, délivrant un message clair : la Rambla del Raval est un espace en pleine transformation, approprié par une politique de réhabilitation qui semble n'oublier personne. Ici, la réalité des mutations du centre de Barcelone, s'impose à l'observateur par l'intermédiaire d'un message immédiatement identifiable: la construction de logements sociaux, sans d'ailleurs évoquer le projet d'hôtel de luxe qui devrait ouvrir ses portes sur le même site.

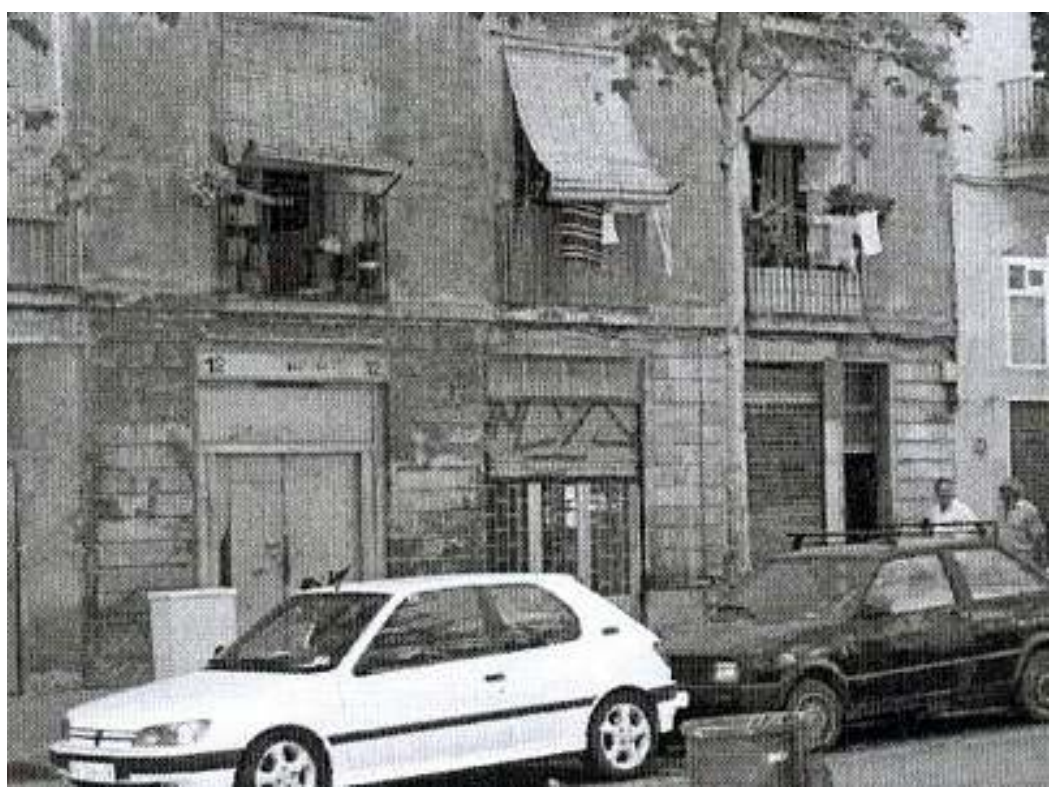


Figure 4. Les laissés-pour-compte de la réhabilitation ? Sept. 2006.

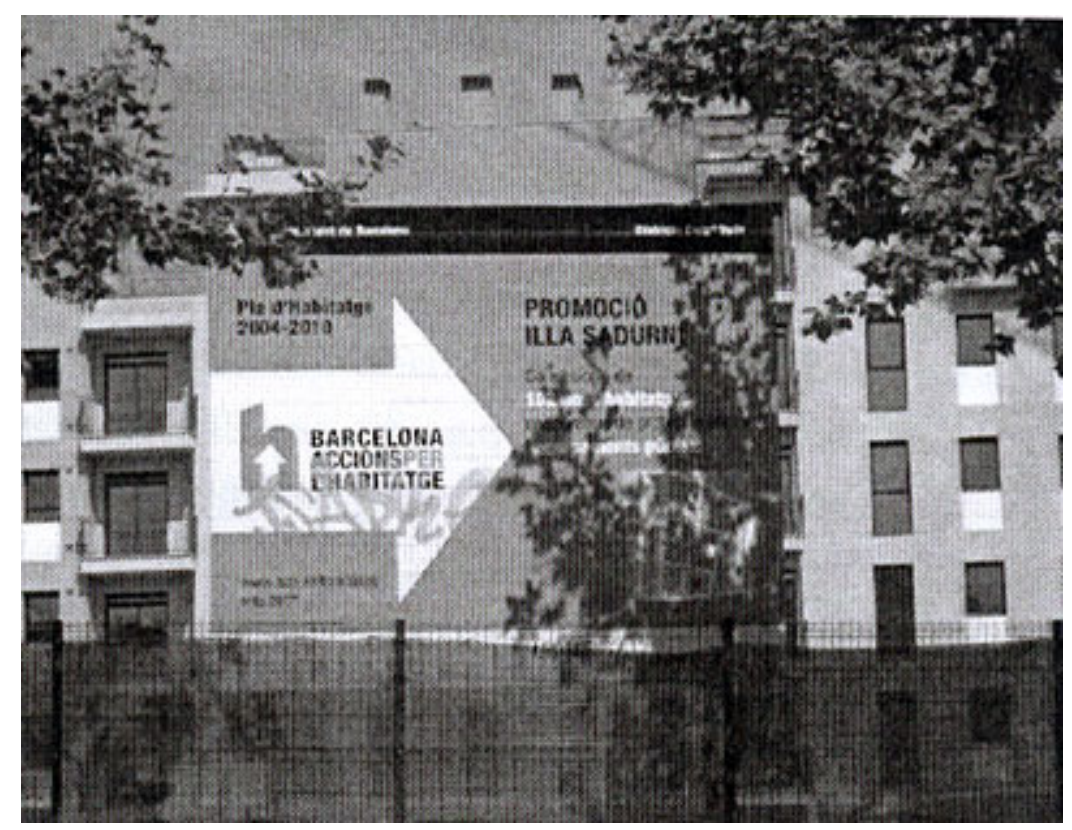

Figure 5. La Rambla del Raval, vitrine de la politique urbaine du centre de Barcelone. Sept. 2006.

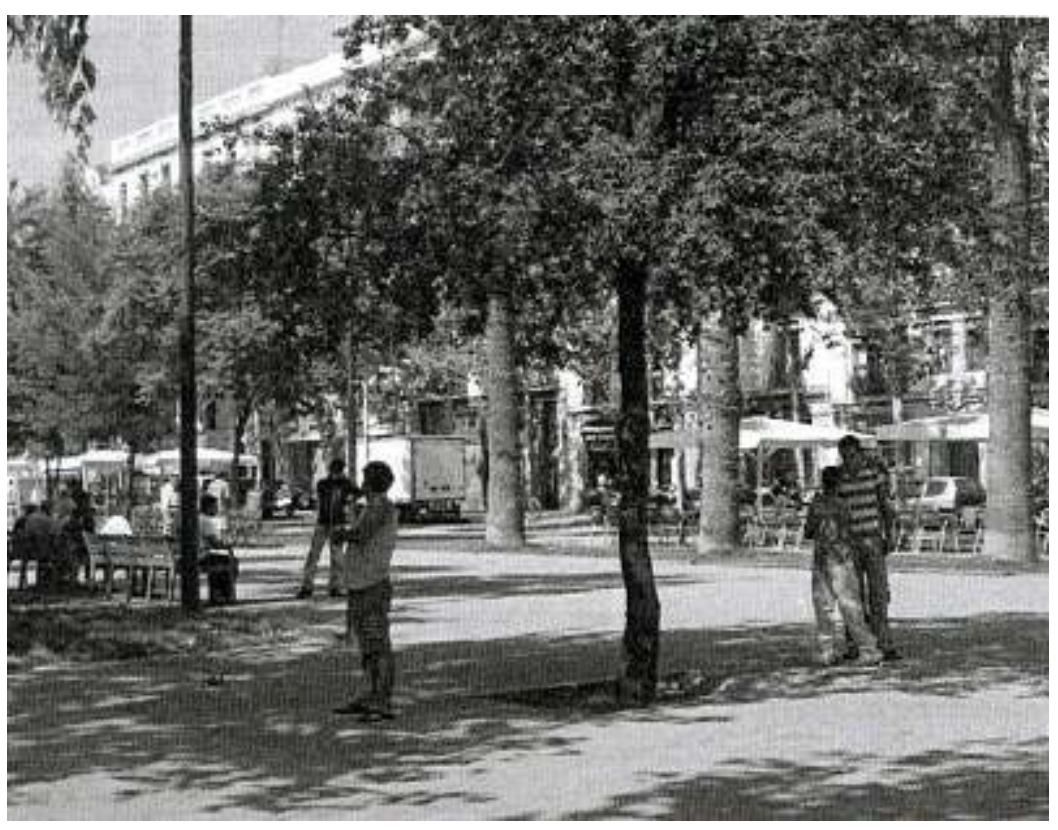

Figure 6. La Rambla del Raval, un espace public. Sept. 2006.

32 La question de la mixité sociale des abords de la Rambla del Raval a déjà été soulevée par la disparité de l'appareil commercial. Prise dans la partie centrale du terre-plein, la figure 6 témoigne du rôle de cet espace particulier comme lieu de promenade et de rencontre. Certains se promènent, d'autres discutent à l'ombre, debout ou sur un banc. En arrière-plan, on distingue deux terrasses de cafés. Cette photographie vise à montrer la pluralité des usages d'un tel espace public, mais également la mixité ethnique qui caractérise le quartier : Latino-Américains (les personnes debout à droite), 
Maghrébins (les personnes âgées sur le banc), Indo-Pakistanais (le jeune homme debout au premier plan)... On remarquera l'absence de femmes. L'espace public serait-il un espace essentiellement masculin ${ }^{35}$ ? Vivant et populaire, le Raval reste un des foyers principaux de l'immigration à Barcelone, une population qui coexiste, parfois à quelques mètres, avec les touristes ou les nouveaux arrivants, comme en témoigne la figure 7.

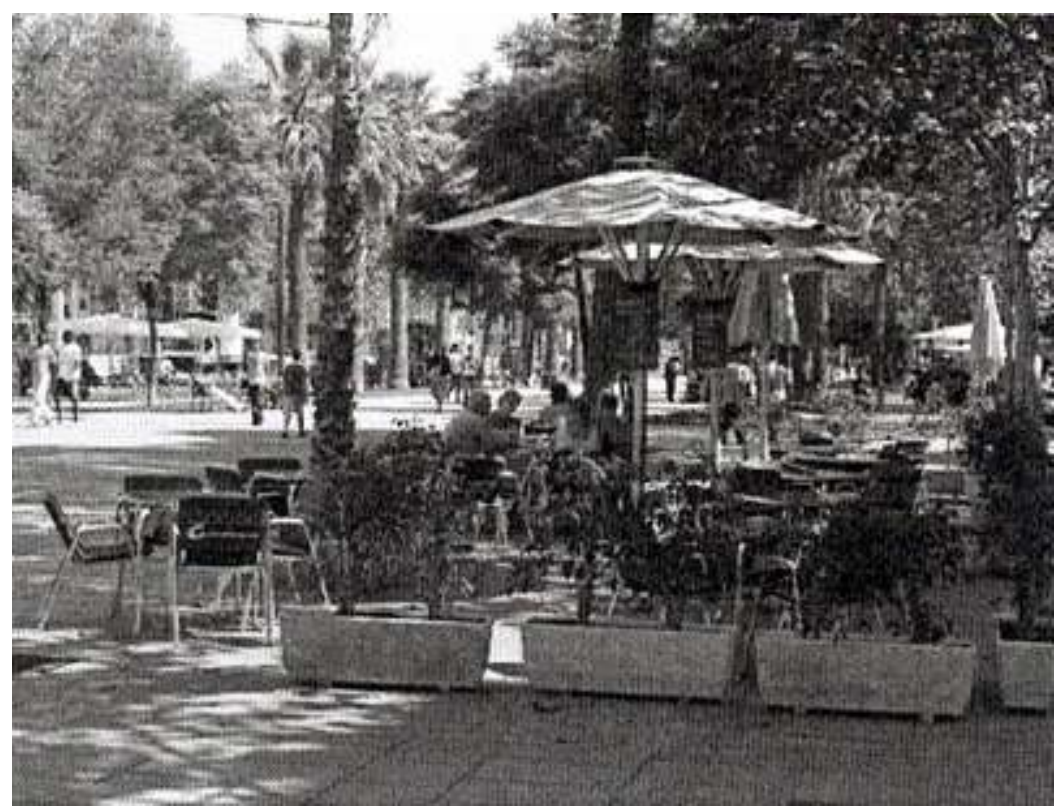

Figure 7. La Rambla del Raval, un espace touristique. Sept. 2006.

La figure 7 peut permettre d'approfondir la réflexion sur la multiplicité des usages de l'espace public, plus difficile à explorer à une échelle si fine par l'approche statistique. Le cadrage met en évidence une terrasse de café relativement grande sur le terre-plein central de la Rambla del Raval. Au milieu des palmiers et des parasols, quelques groupes prennent le frais. En arrière-plan, à gauche, on distingue la terrasse d'un autre café. Les jardinières en pierre sont là comme pour mieux délimiter l'espace public de l'espace réservé à la clientèle du café, à un certain type de population qui ne semble pas se mélanger avec celle qui déambule simplement sur la Rambla. Plus qu'une barrière physique, cette délimitation n'est-elle pas une barrière sociale et symbolique? La population qui fréquente cette terrasse, dans la partie ouest de la Rambla, n'est pas la même que celle du restaurant ou des bancs, vue sur les photos précédentes. Ici, il s'agit plutôt de touristes, reconnaissables par les valises (même si on ne le voit pas sous cet angle ${ }^{36}$ ). On peut donc s'interroger sur la multiplicité des usages de la Rambla del Raval et son attractivité, à la fois potentielle et symbolique, en tant qu'espace public. Qui fréquente ce type d'espace et à quels usages? Y a-t-il complémentarité, conflit ou simplement juxtaposition d'usages par des populations différentes qui ne se mêlent pas, c'est-à-dire les habitants traditionnels du quartier, et les nouveaux usagers du Raval, résidents ou non? Cette question est pertinente dans une réflexion sur la gentrification qui cherche à expliciter le rôle joué par un quartier en plein réinvestissement (à la fois matériel et symbolique). Les cafés témoignent du renouveau que connaît l'activité du Raval, en particulier dans la partie nord, alimenté par de nouvelles pratiques de l'espace tournées vers le tourisme et le loisir, faisant désormais du Raval un quartier «branché ». La Rambla del Raval semble ainsi ici jouer son rôle 
d'espace public à forte mixité sociale. On pourrait émettre l'hypothèse que coexistent autour de la Rambla del Raval trois formes de commerce, donc trois pratiques commerciales différentes : un commerce de proximité, à forte composante migratoire ; un commerce, pas nécessairement de proximité, qui s'adresse à une clientèle plus aisée, qui a les moyens d'investir dans l'immobilier ; enfin un commerce plus touristique mais aussi à destination d'une clientèle locale plus aisée. L'absence d'étude précise sur la clientèle de ces commerces, ne nous autorise pas à affirmer que ces pratiques commerciales témoignent d'une réelle mixité ou sont simplement juxtaposées: les touristes qui se reposent à la terrasse iront-ils ensuite au restaurant halal en contrebas ? Cependant, cette coprésence laisse à penser qu'existent plusieurs usages du quartier, avec notamment une "gentrification de consommation ${ }^{37}$ ", sous l'effet de la proximité des Ramblas ou bien des grands équipements culturels du Raval, une "gentrification résidentielle ", suscitée par la réactivation du marché immobilier dans le centre de Barcelone, sous l'effet de la politique de rénovation urbaine de Ciutat Vella, et des pratiques plus traditionnelles.

Conclusion : apports et limites de la méthode

À l'aide d'un exemple concret, j'ai tenté de montrer en quoi la lecture du paysage urbain pouvait permettre de traiter de la question de la gentrification. Il reste à identifier rapidement quelques apports et limites de cette méthode.

Il convient de souligner que l'analyse du paysage urbain s'appuyant sur un corpus photographique est porteuse de significations. Le choix de la méthode et ses implications (dans la définition du paysage, la composition d'un catalogue photographique et le classement et la mise en ordre de ce dernier) peuvent participer avec profit d'une réflexion sur la gentrification du centre ancien de Barcelone. Dans l'étude de la "Ciutat Vella ", la démarche photographique ne peut constituer l'unique méthode d'analyse mais n'est pas non plus simplement la confirmation ou l'infirmation des hypothèses. Cette approche donne à lire la continuité et les ruptures de l'espace luimême, dans l'étude d'un processus complexe qui touche à la fois les populations et le bâti. La lecture du paysage urbain vise à formuler de nouvelles interrogations, à rendre compte de la réalité des mutations, de la mixité fonctionnelle et sociale, à une échelle micro-urbaine que le découpage statistique traduit imparfaitement. Par l'intermédiaire de ces photos, j'ai ainsi tenté de montrer les mutations profondes du paysage urbain de la Rambla del Raval à la fois dans les pratiques spatiales, les activités et les populations. Le regain d'intérêt par certains types de populations plus aisées laisse supposer une amorce de gentrification, alors qu'au même moment se maintiennent des catégories plus populaires. Il persiste cependant toujours le risque d'interpréter à l'excès les indices photographiques, de donner une vision tronquée de la réalité, d'exagérer certains phénomènes, sous-évaluer d'autres. À mon sens, cet écueil peut être évité dans un travail systématique de repérage et de photographie.

Par ailleurs, l'approche plurisensorielle du paysage paraît essentielle pour traduire des ambiances particulières, des sensations qui donnent sens à la vie de quartier, la pratique de l'espace public, le type d'activités, le type de populations... C'est dans la multiplicité des indices sensoriels que s'appréhende la complexité du tissu urbain et social du centre de Barcelone. Cette dimension du paysage devrait apparaître à travers les commentaires accompagnant les photographies, visant à les réinscrire dans le contexte dans lequel elles ont été prises. 
Enfin, dans la mesure où le travail photographique peut être mené à plusieurs moments, la comparaison des photos doit permettre de rétablir la chronologie et inscrire les mutations du paysage dans le temps ${ }^{38}$. Repérer les différentes étapes de la création de la Rambla del Raval, puis de son appropriation par différents types de population ou d'activités commerciales, c'est mettre en évidence la rapidité de la transformation à la fois sociale, urbaine et économique d'une grande partie du centre ancien de Barcelone. La gentrification est un phénomène qui s'appréhende dans la durée. Pour le comprendre, il appartient d'en saisir les étapes d'évolution et leur traduction dans la continuité du paysage urbain.

\section{NOTES}

1. La thèse, sous la codirection de M. Berger et H. Capel Saez, porte sur « Ciutat Vella à Barcelone : entre réhabilitation et gentrification ", et interroge les mutations sociospatiales du centre ancien de Barcelone dans le cadre des politiques de réhabilitation menées depuis le début des années 1980.

2. Pour une définition plus précise de la gentrification, ses enjeux et les différentes positions au sein du débat, on se réfèrera à : R. Glass, Introduction to London: Aspects of Change, Center for Urban Studies and Mac Gibbon and Kee, London, 1964 ; N. Smith, « Toward a Theory of Gentrification: A Back to the City Movement by Capital not People », Journal of the American Planning Association, $\mathrm{n}^{\circ}$ 45, 1979, p. 538-548 ; D. Ley, "Liberal Ideology and the postindustrial City ", Annals of the Association of the American Geographers, $n^{\circ} 70,1980$, p. 238-258 ; C. Hamnett, « The blind Men and the Elephant: the Explanation of Gentrification ", Transactions of the Institute of British Geographers, vol. 16, $\mathrm{n}^{\circ} 2$ 2, 1991, (traduction française de C. Rhein, « Les Aveugles et l'Éléphant : l'explication de la gentrification », Strates, n 9, 1996-1997, p. 55-79).

3. Notamment, A. Alabart, C. Lopez, «L'Evolució de les Professions dels Barcelonins: gentrificació i polarització ", Barcelona Societat, n 6, 1996, p. 4-18.

4. On trouve quelques références récentes qui témoignent dans un premier temps d'une volonté de définir la gentrification dans son contexte barcelonais. Notamment :

A. Alabart, C. Lopez, loc. cit.; S. Martinez, El Retorn al Centre de la ciutat: la reestructuració del Raval entre la renovació $i$ la gentrificació, thèse sous la direction de C. Carreras, Université de Barcelone, Département de géographie humaine, 2000, 709 p. ; A. Sargatal Bataller, «El Estudio de la Gentrificación », Biblio 3W. Revista Bibliográfica de Geografía y Ciencias Sociales, vol. 5, n 228, 2000 (http://www.ub.es/geocrit/b3w-228.htm);

L. M. Garcia Herrera, « Elitización: Propuesta en español para el término gentrificación », Biblio 3W. Revista Bibliográfica de Geografía y Ciencias Sociales, vol. 6, n 332, 2001 (http://www.ub.es/geocrit/b3w-332.htm) ; G. Vila Bosqued, $L a$ Transformació de la ciutat il el canvi social: els processos de gentrificació a Barcelona, DEA sous la direction d'A. Alabart, Université de Barcelone, 2002, 207 p.

5. A. Berque, Médiance. De milieux en paysages, Tours, Belin, 2000, 156 p.; A. Berque (dir.), Cinq propositions pour une théorie du paysage, Seyssel, Champ Vallon, 1994. 
6. Le terme d'ambiance est pris ici, et dans le reste de l'article, dans son acception la plus neutre, celui d'« atmosphère » (Le Petit Robert, 1983), tout en ayant à l'esprit qu'il renvoie également à une approche particulière de l'espace urbain qui n'est pas sans rapport avec le paysage et qui peut avoir sa place dans l'analyse de la gentrification. 7. S. Rimbert, Les paysages urbains, Paris, Armand Colin, 1973, 236 p.

8. Ibid., p. 16

9. Ibid., p. 16.

10. Ibid., p. 14.

11. Ibid., p. 62.

12. S. Rimbert invite à s'appuyer sur les travaux de C. Jung dans L'homme et les symboles, pour expliciter comment une image particulière de la ville se construit à partir d'une tendance inconsciente.

13. Ces différences semblent apparaitre très clairement dans les premiers entretiens qui ont pu être menés dans le cadre du travail de thèse.

14. D. Mendibil, « Le formatage iconotextuel de l'imagerie géographique des villes », in

F. Pousin (dir.), Figures de la ville et construction des savoirs, Paris, CNRS Éditions, 2005, p. 153.

15. J.-M. Schaeffer, L'image précaire, Paris, Seuil, 1987, 217 p.

16. Ibid., p. 9.

17. À Barcelone, le district est l'équivalent de l'arrondissement. Celui de "Ciutat Vella », qui signifie "Vieille Ville », couvre l'ensemble de la Barcelone médiévale et pré-XIX siècle et compte quatre quartiers : Gótic, Raval, Parc, Barceloneta. 18. Le Raval ne compte plus que 48300 habitants en 1980, contre 105000 en 1960. Voir P. López Sánchez, El Centro histórico: un Lugar para el conflicto. Estrategias del capital para la expulsión del proletario del centro de Barcelona. El caso de Santa Caterina y El Portal Nou, Barcelona, Publicacions i Edicions de la Universitat de Barcelona, Geocrítica, Textos de apoyo, $\mathrm{n}^{\circ} 7,1986,158 \mathrm{p}$.

19. R. Alberch i Fugueras (dir.), Els Barris de Barcelona. Volum I Ciutat Vella, l'Eixample, Barcelona, Enciclopedia catalana, 1999, $389 \mathrm{p}$.

20. Nom donné, en catalan, au quartier de l'Ensanche, construit à la fin du XIX siècle sur les plans de l'ingénieur Cerdá.

21. S. Savary, Imaginaires d'une ville: Barcelone par ses paysages. Une étude géolittéraire, thèse sous la direction d'Y. Luginbühl, Université de Paris-I, 2005.

22. Pour une approche plus complète de la dimension symbolique et mythique du Barrio Chino, qu'on ne peut développer dans le cadre de cet article, on se reportera au travail de S. Savary, op. cit.

23. Notamment le programme "Del Liceu al Seminari », avec la construction du MACBA (Musée d'Art Contemporain de Barcelone) qui a ouvert ses portes en 1995 au cœur du Raval et a contribué à redéfinir la place du quartier dans la géographie culturelle de l'espace urbain.

24. Ce sont des agrégats des sections statistiques.

25. C'est la division statistique de base.

26. Entre 1000 et 2000 familles selon les chiffres officiels, mais près de 4000 selon certaines associations locales de riverains, bien qu'une partie ait été relogée sur place. 27. La Rambla del Raval n'est pas tout à fait orientée nord-sud, mais à Barcelone, pour de nombreux habitants, le bas, la mer, est au sud et le haut, la montagne, est au nord. J'ai repris dans cette analyse cette « orientation fantasmée » qui semble beaucoup plus relever de l'imaginaire collectif que d'un manque de sens de l'orientation. 
28. La Rambla del Raval est devenue, dès 2000 , un lieu privilégié de rassemblement lors des grandes manifestations festives qui rythment l'année barcelonaise du fait de la place disponible.

29. Dans le sens où le bâtiment correspond à certaines normes ou conditions d'habitabilité et de confort.

30. On notera que les Ramblas de Barcelone, les plus célèbres, sont, elles, bordées de platanes, moins exotiques.

31. L'association de riverains El Llar del Avi, située c/ de la Cera.

32. Le fait que la devanture soit écrite en castillan, et non en catalan, révèle sans doute également que les propriétaires du magasin sont eux-mêmes étrangers, du moins non catalans. En l'effet, l'usage à Barcelone est plutôt d'écrire en catalan, et dans un second temps en castillan, ou en anglais (si l'on se trouve proche d'un quartier touristique comme ici).

33. D'autant plus que la photo a été prise après 17 heures, c'est-à-dire après que les commerçants de boutiques non touristiques, qui elles restent majoritairement ouvertes toute la journée, ont achevé leur pause.

34. Habitatges de protecció oficial; ce sont des logements réservés à des ménages à revenus bas ou modérés (jusqu'à 2,5 fois le revenu familial minimum pour les logements en régime spécial, et 5,5 fois pour les logements en régime général). Dans l'état actuel du travail, on ne connaît pas la répartition exacte pour ce projet.

35. On pourrait considérer cette absence de femmes comme le fruit du hasard au moment de la prise de vue. Il convient cependant de noter que pendant les différentes pérégrinations, la population féminine était globalement sous-représentée parmi les personnes présentes.

36. En s'approchant plus près, on a même pu saisir quelques mots d'anglais.

37. Pour reprendre une distinction entre " gentrification de consommation » ou « de fréquentation » et « gentrification résidentielle », opérée par C. Bidou-Zachariasen. Voir C. Bidou-Zachariasen, Retours en ville, Paris, Descartes \& Cie, 2003, 267 p.

38. On pourra à ce titre citer le travail photographique mené par deux artistes barcelonais sur un des édifices qui ont dû être détruits pour laisser place à la Rambla del Raval. Voir I. Banal, J. Canudas, Hospital 106 4t 1a. El Lloc i el temps, 2005.

\section{RÉSUMÉS}

Cet article présente en quoi le paysage urbain permet d'appréhender le phénomène de gentrification. En mettant en évidence la rareté des travaux espagnols sur la gentrification et en s'appuyant sur un corpus photographique de Ciutat Vella (le centre médiéval et pré-XIXe siècle de Barcelone), l'auteur cherche à souligner l'apport de cette méthodologie pour comprendre un phénomène qui modifie en profondeur le paysage urbain et social. L'article s'appuie sur la lecture du paysage urbain élaborée par S. Rimbert et les réflexions de D. Mendibil et J.-M. Schaeffer sur la photographie. Il propose un parcours photographique autour de l'opération de rénovation de la «Rambla del Raval» dans le quartier du Raval (district de Ciutat Vella), spécifique par son histoire, son contexte socio-économique et son rôle dans l'imaginaire collectif à l'échelle de 
l'agglomération. Cette lecture complète l'approche quantitative et met en évidence la juxtaposition de phénomènes contradictoires autour de la Rambla del Raval, entre dégradation et gentrification en cours.

Hovig Ter Minassian, The landscape of gentrification in Barcelona

This article examines the way in which the urban landscape makes it possible to apprehend the phenomenon of gentrification. Pointing to the scarcity of Spanish studies on issues of gentrification and drawing on a corpus of photographs of Ciutat Vella, i.e. the medieval and pre-19th century centre of Barcelona, the author seeks to underline the contribution of this methodology to understand a phenomenon which deeply modifies the urban and social landscape. The article draws on S. Rimbert's conceptualization of urban landscape and on the writings of D. Mendibil and J.-M. Schaeffer on photography. It offers a photographic route around the operation of renovation of the "Rambla del Raval" in the area of the Raval (district of Ciutat Vella), specific by its history, its socio-economic context and its role in the collective imaginary of the whole agglomeration. This methodology is conceived of as complementary to the quantitative approach and highlights the juxtaposition of contradictory phenomena around the "Rambla del Raval", between degradation and gentrification in progress.

\section{INDEX}

Mots-clés : photographie, paysage urbain, centre ancien, Barcelone

Keywords : photography, rénovation, urban landscape, gentrification, perception, Ciutat Vella, Raval, old centre, Barcelona

\section{AUTEUR}

\section{HOVIG TER MINASSIAN}

Géographe, Allocataire-moniteur au Ladyss, (Laboratoire dynamiques sociales et recomposition des espaces), CNRS, 2 rue Valette, 75005 - Paris

hterminassian@laposte.net Toutes les cartes et photos sont de l'auteur. 